\title{
La masculinidad como propaganda de autodefensa estatal en la sociedad patriarcal ${ }^{1}$
}

\author{
Masculinity as state self propaganda patriarchal society
}

\author{
Ddo. Adrián Tarín Sanz \\ Universidad de Sevilla (España) \\ tarinadrian@gmail.com
}

Recibido: 30 de julio de 2013

Aceptado y Publicado: 30 de septiembre de 2013

\section{Resumen}

A pesar de los avances en la cuestión, son todavía numerosos los roles de género que persisten en nuestros imaginarios sociales occidentales. Algunas de estas atribuciones son estratégicamente potenciadas por los poderes hegemónicos tradicionales en sus apéndices de autodefensa: los cuerpos policiales y militares. Valores como la gallardía, la vigorosidad, la resolución o el patriotismo son patrimonio exclusivo del hombre cultural, por lo que para ser un buen soldado al servicio del ciudadano hay que comportarse como tal. La feminidad cobardía, debilidad, homosexualidad o traición- queda ridiculizada hasta el extremo de identificar al enemigo con la mujer cultural. Los individuos y sus diversas orientaciones sexuales y de género deben adulterarse en favor de la salvaguarda del Estado.

\begin{abstract}
Despite advances in the issue, there are still many gender roles that persist in our Western social imaginary. Some of these powers are strategically enhanced by traditional hegemonic powers in self-defense appendices: the police and military. Values such as bravery, the vigor, resolution or patriotism are the exclusive property of cultural man, so to be a good soldier in the service of the citizen to behave as such. Femininity-cowardice, weakness, betrayal is homosexuality or ridiculed to the point of identifying the enemy with cultural woman.

\footnotetext{
${ }^{1}$ Artículo producto de una Ponencia presentada en el VI Encuentro Panamericano de Comunicación COM PANAM 2013 celebrado la Escuela de Ciencias de la Información de la Universidad Nacional de Córdoba (Argentina), durante los días 5, 6 y 7 de junio de 2013.
} 
Individuals and their diverse sexual orientations and gender should be tampered with in favor of the preservation of the state.

Palabras Clave: Propaganda, Masculinidad, Sociedad Patrialcal.

Key Words: Masculinity, propaganda, patriarchal society

\section{Introducción. Propaganda y autodefensa del Estado.}

A lo largo de la Historia, los sistemas de gestión política han demostrado su incapacidad para discurrir inalterablemente con los argumentos de su propio ejercicio, necesitando del enfrentamiento constante para reafirmar su supervivencia, sea éste ejercido como expresión vital y cotidiana o como reacción ante una amenaza. Es en este último caso, cuando se suceden periodos crisis, cuando más evidente se hace esta confrontación, recurriendo el Estado -como símbolo de Poder sistémico, no sólo como órgano político- a la salvaguarda de su ser o de sus intereses. La violencia ejercida como mecanismo de defensa es dual, combinando la de naturaleza directa o física con la simbólica, entendida ésta última por Bourdieu (1993) como una agresión dulce y casi invisible, como un proceso de dominación que "arranca sumisiones que ni siquiera se perciben como tales" (Fernández, 2005, p.9).

El recurso de la violencia discursiva resulta más palpable en las democracias que en los sistemas autoritarios, quizá debido a la certidumbre de que en esa ineludible convivencia entre autoridad y libertad que según Proudhon (1977) toda gobernación padece, el parlamentarismo y la separación de poderes inclina la balanza más hacia el libertarismo. Además, la hegemonía democrática presenta como pilar fundamental el consenso -y no el sometimiento-, por lo que resulta de vital importancia que la dominación parezca $-y$ en ocasiones realmente lo es- un acuerdo entre sistema y componentes.

Tal "irrupción violenta en el mundo de representaciones del prójimo, destinada a privarlo del régimen de su propia conciencia y reducirlo en su condición humana" (Ayala, 1972, p.202), es ejercida habitualmente a través de la propaganda -amén de otros instrumentos propios de la institución imaginaria. Errico Malatesta (1975), con su socorrido estilo panfletario, dio en su momento cuenta del aumento estratégico de la propaganda como sustituto de las porras y las pistolas en un irónico diálogo entre un arquetípico burgués (Ambrosio) y un no menos 
estereotipado juez que conviene en aplicar con dureza la ley. Por su didactismo, resulta muy ilustrativo señalarlo:

Ambrosio. -Ah, sí, eso es verdad. Son necesarias leyes severas y severamente aplicadas. Pero eso no basta. Sólo con la fuerza no se tiene largo tiempo sujeto al pueblo, máxime en los tiempos que corren. Es preciso oponer la propaganda a la propaganda, es preciso persuadir a las gentes de que tenemos razón (p. 125).

Como ya hemos apuntado, la propaganda no es sólo una herramienta sutil de la que se valen las democracias para mantener el orden social desde una postura coherente con el liberalismo político, sino que actúa con la misma determinación para legitimar el sistema a ojos del común ciudadano -y del funcionario que garantiza al Estado su derecho a constituirse como monopolio de la violencia- cuando necesita defenderse de una amenaza o un ataque. De esta manera, este fenómeno comunicativo cumple una doble función: contribuir a la manufacturación del consenso (Lippman, 1991) y tornar admisible la violencia directa (Sierra, 1997). Es esta última aplicación, que tiende a manifestarse únicamente en situaciones preventivas o límites, la que en este trabajo cobra un mayor interés para establecer sus relaciones con la identidad de género.

Una de las situaciones más extremas a las que un Estado ha de enfrentarse es la consumación de un conflicto bélico -ya sea interno o externo, tradicional o asimétrico-, dado que es el momento en que la amenaza se materializa. Los apéndices armados del poder político -el ejército y la policía- cobran su razón de ser y, como apuntábamos anteriormente, se diseñan las operaciones estratégicas de comunicación para hacer aceptable la violencia, algo más difícil de lograr en una sociedad como la democrática, que fomenta un interesado culto al pacifismo. Para ello, la propaganda da a conocer la guerra de manera "aséptica, indeleble y prácticamente higiénica, sin muerte, dolor ni destrucción” (Sierra Caballero, 1997, p.61), impregnando -mejor dicho, pretendiéndolo- a toda una arquitectura de aparatos de transmisión, entre ellos los medios de comunicación. Estos, por intereses económicos, políticos o profesionales condicionan su información en apoyo a alguno de los bandos en conflicto (Maltby \& Keeble, 2007; Schechter, 2004; Wolton, 1992). Así, "los gobiernos imponen controles estrictos sobre lo que se dice y lanzan campañas de propaganda para justificar su posición" bélica, provocando que "hasta los Estados democráticos tengan una regresión autoritaria" (Yehya, 2008, p.53). 
Esta militarización de la comunicación, que se produce en un doble sentido, no sólo porque el estamento castrense pueda tener influencia en los contenidos mediáticos, sino también porque se produce una imitación del lenguaje marcial en los textos periodísticos (Kovacsics, 2007), ha de recurrir al capital simbólico de la sociedad para activarla. Esto es, para persuadir al hombre común de que -metafóricamente- se aliste al ejército y sea capaz de matar al otro (Huici Módenes, 2010). Este material con el que se esculpe la propaganda, procedente del imaginario colectivo, son esquemas de pensamiento que permanecen tanto en la capa visible como en la subterránea del tejido social. Dicho de otro modo: la propaganda no inventa nada, se construye a partir de aquello que ya piensa parte de la sociedad. Garrido Lora (2004) abunda en esta cuestión afirmando que la propaganda de guerra se aprovecha, para cumplir su objetivo, de una sensación de hastío y de miedo preexistente y anterior al propio mensaje, emociones que, si atendemos a una interpretación rigurosa del pensamiento de Marx (2006), podrían estar determinadas por la dinámica del Capital.

En este mismo sentido, otros de los recursos a los que recurre la propaganda de guerra como extensión de la actividad política son la mitología (Huici Módenes, 1996) y los estereotipos, entendiéndolos como "una imagen mental generalmente muy simplificada de alguna categoría de personas, institución o acontecimiento que es compartida en sus características esenciales por un gran número de personas" (Stallybrass, 1977, p. 490). De entre todos los posibles, los roles de género ocupan nuestra cuestión principal al aproximarnos a cómo el Estado los utiliza en su propaganda de guerra para conformar adhesiones y evitar oposiciones empleando cualquier instrumento a su alcance, como los media.

\section{Los roles de género como alimento de la propaganda de guerra}

Resulta pertinente realizar una primera aproximación a los roles de género, a su naturaleza y a sus características. Uno de los conceptos con el que mantienen un estrecho vínculo y por el que se ven -recíprocamente- afectados es la noción de identidad de género, entendida como:

an individual's self-conception as being male or female, as distinguished from actual biological sex. (...) Gender identity is not fixed at birth; both physiologic and social 
factors contribute to the early establishment of a core identity, which is modified and expanded (Gender identity, 2013).

A la vista de la definición, y puesto que toda identidad supone "un proceso por el cual el sujeto se constituye y se transforma, asimilando o apropiándose de aspectos, atributos 0 rasgos de lo que lo rodean" (Frigerio, 2004, p.147), podemos afirmar que la autoconcepción de género es relacional; un constructo social cuya consciencia también es individual. La identidad de género, por tanto, está constituida por una numerosa gama de concepciones e interacciones materiales y simbólicas, por "un conjunto de acuerdos tácitos o explícitos elaborados por una comunidad y que influye en los procesos de enseñanza-aprendizaje", generando una "asignación social de funciones y actividades a las mujeres y a los hombres" (Rodríguez Wangüemert, Matud Aznar \& Espinosa, 2008) denominadas roles de género. Estas expectativas colectivas de comportamiento atribuidas a lo masculino y lo femenino, compartidos en mayor o menor medida por segmentos de la sociedad, son reproducidas en la propaganda de guerra al exaltar u ocultar algunos de sus valores comúnmente asociados.

Tanto la identidad como los roles de género están determinados sustancialmente por la cultura en la que se desarrollan. Estas esperanzas y conceptualizaciones sociales de cómo deben ser y actuar los hombres y las mujeres no se dan en el vacío, sino que actúan en el marco de "un sistema de relaciones sociales sexo-políticas" instituido "por los varones, quienes como grupo social y en forma individual y colectiva oprimen a las mujeres" y "se apropian de su fuerza productiva y reproductiva, de sus cuerpos y sus productos, ya sea con medios pacíficos o mediante el uso de la violencia" (Fontenla, 2008). Este sistema cultural, habitualmente denominado patriarcado, determina el material simbólico al que recurrimos para establecer los actuales roles de género. Quizá pueda pensarse, y se tendrá en ello parte de razón, que el hombre en su situación de privilegio patriarcal sólo puede obtener beneficios del ejercicio de su dominio. No obstante, tal supremacía, aunque a corto plazo pueda resultar ventajosa para el poderoso y dañina para el desposeído, es un fenómeno maligno y bifocal, ya que el establecimiento de roles se comparte por y para ambos géneros, empleando una simplificación -y, por tanto, adulteración- inherente al estereotipo:

A pesar de que vivimos en una cultura de estereotipos sexistas, en los que aparentemente se privilegia al hombre sobre la mujer, en realidad los estereotipos afectan negativamente a ambos géneros, al constituirse en paradigmas divisionistas 
que no permiten el descubrimiento, el desarrollo y la expresión de cualidades y valores propios del ser humano, sin distinción de sexo (Suárez, 2006, p.12).

Aunque actualmente existe una tendencia prolífica y de interés acerca de la construcción de una nueva masculinidad positiva -creadora, innovadora- (Moore \& Gillette, 1993) o difusa androginia- (Pérez Rojas \& Mesa Franco, 2008), otros autores y autoras todavía observan en la sociedad occidental contemporánea valores y roles tradicionales asociados a lo masculino. Algunos de ellos son útiles para el discurso propagandístico del Estado cuando pretende anular a su oposición; tal es el caso de la fortaleza masculina como atractivo sexual:

El modelo masculino de belleza sigue siendo para la mayoría de las mujeres el hombre vigoroso con fuertes músculos, capaz de efectuar hazañas en la que deba ser empleada la fuerza bruta (...). Esto no sería tan preocupante si no fuera acompañado por el convencimiento de que la fuerza bruta, la violencia es el modo en que deben ser resueltos los conflictos. Los chicos admiten que es lo que tienen que hacer y las chicas admiran que lo hagan, a veces a costa de indeseables consecuencias y sufrimientos para unas y para otros (Toajas Roger, 2012, p. 367).

Que los hombres -como sujetos de quienes se espera que se adecúen a los roles masculinos- convivan en una cultura patriarcal y heteronormativa donde se prestigia el empleo de la violencia física como acto de seducción afectiva, allana el terreno a la propaganda para disculpar la guerra o persuadir a la población para que ingresen en los cuerpos de seguridad del Estado.

Esta inclinación del hombre por la violencia es argumentada según algunas investigaciones en base a cuestiones biológicas, considerando innata su agresividad "porque es verdad que la experiencia de la vida cotidiana evidencia claramente que los niños son más activos y practican juegos más violentos que las niñas" (Huici Módenes, 2010, p. 168). Una violencia exhibicionista, ya que "es normal que, al igual que ocurre con otros animales superiores, el hombre entre en competencia con otros en la demostración de su virilidad, su fuerza y su poder" (Garrido Lora, 2004, p.120). Sin embargo, y como apuntamos desde el comienzo en el presente artículo, "sin desdeñar la base biológica, los estudios más recientes demuestran que no se puede hablar de una masculinidad única", por lo que "buena parte de lo que hoy consideramos virilidad, masculinidad, hombría, etc., es una construcción cultural" (Huici 
Módenes, 2010, p.169). En esta línea apunta Garrido Lora (2004) al afirmar que la masculinidad, el machismo, la hombría, el honor y la competitividad, principios atribuidos al hombre en la cultura patriarcal, son aludidos frecuentemente en la propaganda hegemónica para estigmatizar socialmente a quienes osen no suscribirse a la tesis militarista. Así, la propaganda, como fenómeno comunicativo simplificador que es y que reduce la existencia a la dicotomía teológica del Bien y del Mal, opone la feminidad a la masculinidad. Una mujer sólo podrá ser valiente y gallarda, y por tanto sólo podrá defender al Estado, si adopta roles de comportamiento masculinos, perdiendo así su identidad de género. Son, como afirma Héritier, "mujeres con corazón de hombre", que "no se comportan con la reserva y la modestia propias de su sexo [sic], sino con agresividad, arrogancia y audacia" (2002, p.224). Frente a la incontestable realidad de que el estamento militar o policial en su escalafón inferior requiere de la fuerza bruta, y que el hecho de que haya mujeres que la empleen no tiene por qué deberse a una dominación de género sino a una cuestión operativa, parece acertado afirmar que para la propaganda $-\mathrm{y}$ por ende en parte del imaginario socialcomportarse como un soldado es sinónimo de comportarse como un hombre, cobrando mayor importancia la interpretación del hecho social que el propio hecho en sí.

\section{Aplicaciones de la masculinidad en la propaganda de guerra: muestras concretas}

Como hemos apuntado anteriormente, la agresividad y la fortaleza física pertenecen al ámbito de la masculinidad y su actividad se reserva tradicionalmente a los hombres, hecho que ha tenido un impacto histórico notable en la defensa del Estado. Aunque actualmente la mayor parte de los ejércitos admiten mujeres entre sus filas, podemos considerar esta integración como un fenómeno reciente: hace tan sólo veinticinco años que las mujeres del Estado español pueden formar parte del estamento militar, una cifra que se antoja minúscula en comparación con la trayectoria vital de los ejércitos. Asimismo, no ha sido hasta comienzos de 2013 que Estados Unidos, uno de los países de referencia para el mundo occidental, revocó la normativa que impedía a las mujeres participar en acciones terrestres de combate. No obstante, esta nueva política norteamericana no obedece a un cambio de paradigma castrense ni posee una correspondencia con el sentir militar, sino con ciertos sectores liberales de la sociedad. Amén de las argumentadas razones físicas -una pretendida menor fortaleza de la mujer- y psicológicas -según los opositores a la derogación las mujeres tienden a sufrir conflictos post-traumáticos con más frecuencia que los hombres-, 
existe una desconfianza latente de los soldados hombres hacia las mujeres al considerarlas inútiles para el combate (Browne, 2007).

Esta supuesta ineficacia que manifestarían las mujeres en situaciones estresantes y violentas se traslada por asociación hacia la homosexualidad en los hombres. En diciembre de 2010, el gobierno de los Estados Unidos derogó la ley Don't ask, don't tell, según la cual se sancionaba a los miembros de las Fuerzas Armadas que expresaban o manifestaban una orientación sexual diferente a la heteronormatividad. Esta legislación, introducida por Bill Clinton en 1993, aunque resulte paradójico atendió a una pretendida integración de la homosexualidad y la bisexualidad en el ejército, ya que anteriormente el estamento militar norteamericano se encontraba reservado para los y las heterosexuales. Al igual que la incorporación de la mujer a las operaciones de combate, entre los soldados estadounidenses la abolición de la ley Don't ask, don't tell tuvo una acogida negativa (Zogby International, 2006). Algunos de los argumentos que justifican la oposición a aceptar la homosexualidad en el ejército son coherentes con los esgrimidos para impedir que las mujeres pertenezcan a unidades de combate terrestres: desconfianza en sus capacidades militares y malestar ante una posible tensión sexual que envilezca la convivencia del grupo (Bailey \& Barbato, 2008). Como afirma Badinter, "en el lenguaje cotidiano, el homosexual no es exactamente el hombre que mantiene relaciones sexuales con otro hombre, sino el que se supone que adopta un papel pasivo: el homosexual es, en realidad, marica, plumón, loca... una mujer, en definitiva" (1993, p.144). El lenguaje, como expresión verbal del imaginario social, muestra la estrecha relación existente entre homosexualidad y feminidad, contagiando parte del estereotipo femenino y potenciando, por oposición, los mecanismos de promoción de la masculinidad en la autodefensa del Estado.

Pero no sólo puede deducirse la exaltación de los roles de género masculinos y la minusvaloración de los femeninos a través de la legislación, los reglamentos internos o los imaginarios sociales del ejército, elementos que, a fin de cuentas, no son sino la expresión de unos esquemas de pensamiento determinados, sino que también los propios contenidos concebidos intencionalmente como propagandísticos -aquellos a los que Pizarroso (1999) atribuye un animus propagandi- reproducen los valores asociados a la masculinidad. En 2010 el ejército austríaco tuvo que retirar un spot televisivo de reclutamiento ante la presión de diferentes organizaciones feministas. En el anuncio, un hombre caracterizado con una estética villanesca se ofrecía a varias mujeres adolescentes utilizando como reclamo un vehículo deportivo; ante la incomodidad de la situación, de entre la niebla surgió un tanque 
del ejército austríaco con un apuesto soldado sobre la escotilla que, tras acariciar con insinuaciones fálicas el cañón del vehículo, consigue rescatarlas. La imagen estereotipada de la mujer desvalida y el hombre protector subyacía en tal mensaje propagandístico.

\section{Conclusiones}

La masculinidad, como constructo social, está en evolución continua, aunque ésta se produzca a través de un goteo tenaz al cambio. Esa resistencia, ese reflejo en las estructuras estáticas del pasado, desprende un ideal de hombre -pues la heteronormatividad hegemónica todavía no subvertida identifica el rol de género con el sexo biológico- que puede ser, y es, aprovechado por la propaganda estatal para activar un determinado comportamiento en todos/as aquellos y aquellas que deseen o se vean obligados a defender el statu quo. Las actitudes a las que nos referimos, como la virilidad, la valentía o la agresividad, se convierten en valores medulares de los cuerpos policiales y militares; así, tanto los hombres como las mujeres que se alisten deben pervertir su propia identidad (de género) en caso de que no se adecúen al esquema simbólico impuesto, al no haber apenas espacio para la feminidad o para otros patrones o personalidades.

De igual manera, la situación de privilegio de la masculinidad es, a su vez y aunque resulte un oxímoron, material e ilusoria. $Y$ lo es porque al mismo tiempo que se prestigia comportarse como un hombre, ello no es intrínsecamente beneficioso:

"[A los hombres] Se les embrutece, se les mina la moral para que sean buenos soldados y buenos trabajadores. El día en que los hombres vean claro cómo el Estado y el capitalismo han usado y abusado de su masculinidad y cómo los han dejado en muchos casos mutilados moral y emocionalmente, el día en que los hombres hagan el camino de desvelar sus opresiones de género, cómo hemos hecho nosotras, ese día, llorarán de pena, asco y vergüenza" (Hernández Catalán, 2011, p.32).

Exceptuar un ámbito laboral a un determinado conjunto de roles de género no sólo supone la perpetuación de una determinada cultura (la patriarcal) o la concreción de una pretendida homogeneidad operativa, sino que también se transforma en un mecanismo de control poblacional conveniente para el Estado. Las disfunciones arbitrarias basadas en un exceso de violencia física pueden ser disculpadas en base a la propia naturaleza de los organismos de Defensa y comprendidas, por tanto, por la sociedad. De esta manera, acostumbrar a la población a que (1) la defensa del Estado siempre es necesaria y que (2) su violencia es 
aceptable porque (3) tal es el estado de cosas, y que (4) quien disienta es enemigo y cobarde -y es equiparado/a a una mujer-, es uno de los actuales triunfos del Poder.

\section{Bibliografía}

AYALA, F. (1972). Hoy ya es ayer. Madrid: Moneda y Crédito.

BADINTER, E. (1993). XY. La identidad masculina. Madrid: Alianza Editorial.

BAILEY, F. \& BARBATO, R. (2008). The strange story of Don't ask, don't tell. HBO.

BROWNE, K. (2007). Co-ed Combat: The New Evidence that Women Shouldn't Fight the Nation's Wars. South Florida: Sentinel.

BOURDIEU, P. (1993). "La violenza simbolica". Enciclopedia Multimediale delle Scienze Filosofiche. Consultado el 11 de abril de 2013 en la WWW: http://www.emsf.rai.it/interviste/interviste.asp?d=388

FERNÁNDEZ, J.M. (2005). "La noción de violencia simbólica en la obra de Pierre Bourdieu: una aproximación crítica". Cuadernos de Trabajo Social, vol. 18. Madrid: Universidad Complutense.

FONTENLA, M. (2008). “¿Qué es el patriarcado?”, en GAMBA, S. (Ed.). Diccionario de estudios de género y feminismos. Buenos Aires: Biblos. Consultado el 12 de abril de 2013 en la WWW: http://www.nodo50.org/mujeresred/spip.php?article1396.

FRIGERIO, G. (2004). "Identidad es el otro nombre de la alteridad. La habilitación de la oportunidad". Revista Ensayos y Experiencias № 52. Buenos Aires: Noveduc.

GARRIDO LORA, M. (2004). “¿Qué valores humanos utiliza la propaganda de guerra en los conflictos?”; en HUICI MÓDENES, A. (ed.). Los heraldos de acero. La propaganda de guerra y sus medios. Sevilla: Comunicación Social Ediciones y Publicaciones.

"Gender identity" (2013). En Encyclopedia Britannica. Consultado el 11 de abril de 2013 en la WWW: http://global.britannica.com/EBchecked/topic/228219/gender-identity

HÉRITIER, F. (2002). Masculino / Femenino. El pensamiento de la diferencia. Barcelona: Ariel. 
HERNÁNDEZ CATALÁN, R. (2011). Feminismo para no feministas. Madrid: Federación de Mujeres Jóvenes.

HUICI MÓDENES, A. (1996). Estrategias de la persuasión. Mito y propaganda política. Sevilla: Alfar.

HUICI MÓDENES, A. (2010). Guerra y propaganda en el siglo XXI. Nuevos mensajes, viejas guerras. Sevilla: Alfar.

KOVACSICS, A. (2007). Guerra y lenguaje. Barcelona: Acantilado.

LIPPMAN, W. (1991). Public Opinion. New Jersey: Transaction Publishers.

MALATESTA, E. (1975). Socialismo y anarquía. Madrid: Ayuso.

MALTBY, S. \& KEEBLE, R. (2007). Communicating war. Memory, media and military. Londres: Arima Publishing.

MARX, K. (2006). Manuscritos económico-filosóficos de 1844. Buenos Aires: Ediciones Colihue.

MOORE, R. \& GILLETTE, D. (1993). La nueva masculinidad. Rey, guerrero, mago y amante. Barcelona: Paidós.

PÉREZ ROJAS, L.B. \& MESA FRANCO, A.L. (2008). "Andros y gyne. Lo inevitable del nuevo milenio". Revista CES Psicología, vol. 1, no 2. pp. 83-92. Medellín: Universidad CES.

PIZARROSO, A. (1999). "La historia de la propaganda: una aproximación metodológica". Historia y comunicación social, no4. pp.145-172. Madrid: Universidad Complutense de Madrid.

PROUDHON, P.J. (1977). El principio federativo. Madrid: Editora Nacional.

RODRÍGUEZ WANGÜEMERT, C., MATUD AZNAR, P. \& ESPINOSA, I. (2008). "Roles de género en la prensa diaria nacional". Estudios sobre el mensaje periodístico, 14. pp. 575580.

SCHECHTER, D. (2004). Las noticias en tiempos de guerra. Medios de comunicación, ¿información o propaganda? Barcelona: Paidós.

SIERRA CABALLERO, F. (1997). Comunicación e insurgencia. Hondarrubia: Hiru.

STALLYBRASS, O. (1977). "Stereotype"; en BULLOCK, A. \& STALLYBRASS, O. (dirs.). The Fontana Dictionary of a Modern Tough. Londres: Fontana/Collins. 
SUÁREZ, J.C. (2006). La mujer construida. Comunicación e identidad femenina. Sevilla: Editorial Mad.

TOAJAS ROGER, M.V. (2012). "Amor y violencia en el espacio afectivo". Actas I Congreso Internacional de Comunicación y Género. Sevilla: MAD.

WOLTON, D. (1992). War Game. La información y la guerra. Madrid: Siglo XXI.

YEHYA, N. (2008). Guerra y propaganda. Medios masivos y mito bélico en Estados Unidos. Barcelona: Paidós.

ZOGBY INTERNATIONAL (2006). Opinions of Military Personnel on Sexual Minorities in the Military. Consultado el 18 de abril de 2013 en la WWW: http://www.palmcenter.org/files/active/0/ZogbyReport.pdf

\section{Forma de citar este artículo en bibliografías}

Tarín Sanz, A. (2013): "La masculinidad como propaganda de autodefensa estatal en la sociedad patriarcal", en Revista PANGEA, 4, páginas 149 a 160. Red Académica Iberoamericana de Comunicación. Recuperado el __ de ___ de 2 __ de: http://www.revistapangea.org 